\title{
Investigation of severity of voltage flicker caused by second harmonic
}

\begin{abstract}
Second harmonic produces asymmetrical waveform which leads to fluctuation of root-meanssquare (rms) value with certain frequency. Meanwhile, function of weighting filter in flickermeter is designed for sixth-order low-pass filtering function with frequency to be limited to $35 \mathrm{~Hz}$, signal with frequency ranging from 35 to $100 \mathrm{~Hz}$, at certain gain, still can pass through. Since the second harmonic produces fluctuation of rms value with $50 \mathrm{~Hz}$, there is high possibility that it can be passed by this filter and thus resulting in flicker detection. Therefore, this study points out a worthwhile research gap to justify that the presence of the second harmonic can directly increase severity of voltage flicker. In addition, further simulation work using semi-converter has been carried out, as one of main even harmonic sources, in order to justify the concept. To practically validate the concept, Fluke power analyser 435 series II and programmable alternative current source are utilised in the experimental setup. In the main finding, it is confirmed that severity of the voltage flicker increases directly with the presence of the second harmonic and it also formed a clear relationship between short-term perceptibility (Pst) and percentage of the second harmonic component.
\end{abstract}

Keyword: Voltage flicker; Second harmonic; Weighting filter; Flickermeter 